\title{
ESTUDO DESCRITIVO DE ALTERAÇÕES POSTURAIS SAGITAIS DA COLUNA LOMBAR EM ESCOLARES DA REDE FEDERAL DE ENSINO DE FLORIANÓPOLIS
}

\author{
DESCRIPTIVE STUDY OF SAGITTAL LUMBAR SPINE CHANGES IN STUDENTS \\ OF THE FEDERAL EDUCATIONAL SYSTEM OF FLORIANÓPOLIS
}

Susane Graup', Saray Giovana dos Santos², Antônio Renato Pereira Moro 2,3

\section{RESUMO}

Objetivo: O objetivo deste estudo foi analisar a prevalência de alterações posturais sagitais na coluna lombar e fatores associados em escolares da Rede Federal de Ensino de Florianópolis. Métodos: Foram avaliados 288 adolescentes (156 do sexo masculino e 132 do feminino), na faixa etária dos 15 aos 18 anos, estudantes da Rede Federal de Ensino de Florianópolis/ SC. Para o levantamento dos dados utilizou-se: fotogrametria seguindo protocolo específico para avaliação postural e questionário para identificar a prevalência de quadros de dor, frequência e atividades que desencadeavam o problema. $\mathrm{Na}$ análise de dados utilizou-se de estatística descritiva, teste t de Student para amostras independentes, Qui-quadrado e regressão de Poisson, adotando a significância de 0,05 em todos os testes. Resultados: A prevalência de dor lombar foi de $49,3 \%$, apresentando uma frequência semanal em $43,1 \%$ dos avaliados. A prevalência de desvios foi de $53,8 \%$, sendo que $90,9 \%$ corresponderam à retificação da curvatura lombar, acometendo mais o sexo masculino. Foi encontrada diferença significativa no ângulo lombar entre os sexos, sendo que o grupo masculino apresentou razões de prevalência superiores de desvios posturais. A variável dor lombar não apresentou associação com desvios posturais sagitais. Conclusão: As prevalências de dor e desvios posturais na coluna lombar foram elevadas, sendo que a relação entre essas variáveis não se apresentou de forma significativa. As principais causas de dor lombar foram a prática de esportes ou atividades vigorosas e a permanência durante longos períodos de tempo na posição sentada.

Descritores - Coluna Vertebral; Dor Lombar; Postura; Adolescentes

\section{ABSTRACT}

Objective: The purpose of this study was to assess the prevalence of sagittal lumbar spine changes and associated factors in students of the federal educational system of Florianópolis. Methods: 288 adolescents (156 males and 132 females), aged from 15 to 18 years, students of the Florianópolis' federal school took part in this study. Photometry was used following a specific protocol for postural assessment, and a questionnaire was used to identify the prevalence and frequency of low back pain, and activities that triggered the problem. Descriptive statistics, Student's t-test for independent samples, chi-square test and Poisson's regression were used, with the level of significance set at $p<0.05$. Results: The prevalence of low back pain was $49.3 \%$, with weekly frequency in $43.1 \%$ of the sample. The prevalence of deviation was of $53.8 \%$, and 90.9\% corresponded to the rectification of the lumbar curve, affecting more males. Significant differences in lumbar angle between the sexes were found; higher prevalence ratios of lumbar postural deviations was found in the male group. Conclusions: The prevalences of low back pain and lumbar postural changes in adolescents were high, and no significant relationship between those variables was found. Practicing sports or vigorous activities and remaining in a seated position for extended periods of time were the principal causes of lumbar pain.

Keywords - Spine; Low Back Pain; Posture; Adolescents

1 - Aluna do Programa de Pós-Graduação em Engenharia de Produção da Universidade Federal de Santa Catarina - UFSC - Florianópolis (SC), Brasil.

2 - Professor do Departamento de Educação Física da Universidade Federal de Santa Catarina - UFSC - Florianópolis (SC), Brasil.

3 - Professor do Programa de Pós-Graduação em Engenharia de Produção da Universidade Federal de Santa Catarina - UFSC - Florianópolis (SC), Brasil.

Trabalho realizado no Laboratório de Biomecânica da Universidade Federal de Santa Catarina, Florianópolis, SC.

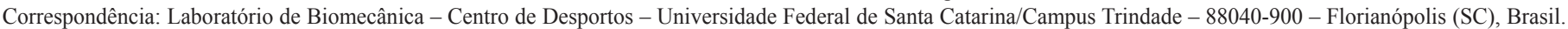

E-mail: sg_ef@hotmail.com 


\section{INTRODUÇÃO}

A dor na região lombar ou lombalgia, como é chamada, tem sido considerada uma causa frequente de morbidades e incapacidades na população em geral; no entanto, sua causa nem sempre é específica ${ }^{(1)}$. Independentemente das causas, a dor lombar atinge níveis epidêmicos, acometendo de 70 a $85 \%$ dos indivíduos ao menos uma vez na vida ${ }^{(2)}$.

Inúmeras são as circunstâncias que contribuem para o desencadeamento de dor lombar, sendo considerada por alguns autores como uma doença multifatorial ${ }^{(3,4)}$. A etiologia da dor lombar é difícil de ser identificada pelo fato de se manifestar sob várias condições. Dentre outras causas, as dores têm apresentado associação com fatores como: sexo feminino ${ }^{(1,3,5,6)}$, obesidade $^{(1,6)}$, sedentarismo ${ }^{(6)}$, níveis elevados de atividade física $^{(1,3,5,7)}$, flexibilidade reduzida ${ }^{(4)}$ e hábitos posturais $^{(6,8,9)}$.

Mesmo com alguns estudos comprovando a associação entre desvios posturais e dor lombar ${ }^{(10,11)}$, essa relação não é consenso na literatura ${ }^{(1)}$. Entretanto, mudanças no padrão postural são apontadas como fator de risco para o desenvolvimento de dores na lombar ${ }^{(9)}$, pois a postura anormal causa uma tensão nos ligamentos e músculos que indiretamente afetam a curvatura lombar, desencadeando dor.

$\mathrm{Na}$ adolescência, a prevalência de dor lombar vem aumentando consideravelmente nas últimas décadas ${ }^{(12)}$, sendo essa problemática ainda mais significativa quando se perpetua para a idade adulta ${ }^{(2)}$. Nessa perspectiva, o estudo de revisão de Balagué et al ${ }^{(1)}$ sobre dor lombar em adolescentes, apontou prevalências elevadas, variando entre 30 e $51 \%$.

Não obstante a isso, os casos de alterações posturais da coluna vertebral na adolescência vêm crescendo consideravelmente, por ser uma faixa etária em que o indivíduo está exposto a sobrecargas crescentes, tais como o suporte de mochilas escolares de maneira assimétrica e inadequada ${ }^{(13)}$ ou ainda a permanência na posição sentada, adotando posturas indevidas por longos períodos de tempo $^{(14)}$.

Deste modo, com o intuito de contribuir com a literatura da área e, principalmente, para poder posteriormente intervir, é que este estudo foi elaborado com o objetivo de analisar a prevalência de alterações posturais sagitais na coluna lombar e fatores associados em adolescentes escolares da Rede Federal de Ensino de Florianópolis.

\section{MÉTODOS}

Foi realizado um estudo descritivo transversal com adolescentes de 15 a 18 anos das escolas de ensino médio da Rede Federal de Ensino de Florianópolis, SC. A população foi estimada em 850 alunos, nessa faixa etária, matriculados em duas escolas.

Para o cálculo da representatividade amostral foi utilizada a equação proposta por Rodrigues ${ }^{(15)}$, que estabelece o tamanho da amostra para variáveis quantitativas, considerando um erro amostral de 5\% e nível de confiança de $95 \%$. Dessa forma, o número de indivíduos da amostra calculada foi de 265 adolescentes. Como as escolas localizavam-se na mesma região geográfica, não houve necessidade de estratificar por localidade. No entanto, a amostra foi estratificada por gênero, sendo realizado um sorteio simples ao acaso dentro dos estratos.

Como critérios de exclusão foram adotados: recusa do escolar em participar do estudo, não entregar o Termo de Consentimento Livre e Esclarecido assinado pelos pais ou responsáveis, não estar na faixa etária do estudo, não realizar um dos testes necessários para as análises e/ou apresentar deficiências físicas que impossibilitassem a realização de qualquer um dos procedimentos necessários para atingir os objetivos do estudo. Sendo assim, a amostra foi constituída por 288 alunos, sendo 156 do sexo masculino e 132 do feminino, com média de idade de $16 \pm 1,06$ anos e 15,87 $\pm 1,05$ anos, respectivamente.

Todos os protocolos e procedimentos adotados no estudo foram aprovados pelo Comitê de Ética e Pesquisa com Seres Humanos da Universidade Federal de Santa Catarina, sob o protocolo n ${ }^{\circ} 048 / 07$, que acompanha a resolução 196/96 do Conselho Nacional de Saúde

A obtenção dos dados relativos à avaliação postural da coluna lombar, seguiram os procedimentos de Christie et $a l^{(10)}$, que demonstraram alta correlação com dados radiográficos com nível de significância de 0,01 . O ângulo da lordose lombar foi quantificado na posição ortostática, no plano sagital direito, no qual os processos espinhosos das vértebras T12 (12ª vértebra torácica) e L5 (quinta vértebra lombar) foram identificados e demarcados sobre a pele por meio de palpação, sendo aderidos nestes pontos hastes de $7 \mathrm{~cm}$ de comprimento e de peso desprezível (3,4 gramas), com base redonda, levemente côncava, possuindo $1 \mathrm{~cm}$ de raio, para permitir a fixação sobre os processos transversos com fitas adesivas. 
Estas hastes permitiram projetar os pontos anatômicos no plano sagital a fim de evitar que as protuberâncias musculares dificultassem a visualização das marcas. O ângulo da lordose lombar foi definido pela intersecção do prolongamento das hastes posicionadas sobre os processos espinhosos das vértebras T12 e L5.

Para a análise dos desvios sagitais da lombar foram utilizadas imagens fotográficas de uma máquina digital com resolução de 3.0 megapixels, que permitiram posterior digitalização em um programa com software gráfico que possibilitou identificar a angulação gerada pela intersecção dos pontos. Para registro da fotografia, inicialmente calibrou-se um ponto fixo suspenso em um fio de prumo padronizado. A câmera foi posicionada sobre um tripé a 1,20 metros de altura do solo e a três metros dos avaliados. O alinhamento postural da coluna lombar foi classificado como normal, diminuição do ângulo lombar (retificação) e acentuação do ângulo lombar (hiperlordose) através da análise dos valores angulares gerados, considerando como normais os valores de $30-45^{\circ}$.

As informações referentes à dor lombar foram obtidas por meio de um questionário construído, composto por uma questão fechada e duas abertas, as quais questionavam sobre a incidência, frequência e atividades que geravam dores na coluna lombar. A clareza das questões foi testada com 10 sujeitos com as mesmas características da amostra estudada, obtendo-se um índice de $96 \% \mathrm{e}$ a validade de conteúdo com cinco especialistas obtendo um índice de $93 \%$.

Para o tratamento dos dados foi utilizado o programa SPSS (Statistical Package for the Social Sciences) para Windows versão 15.0. Primeiramente, foi realizada uma estatística descritiva dos dados, amparada por dados organizados através de tabelas de distribuição de frequência, porcentagem e medidas de tendência central (média) e de dispersão (desvio padrão). Foi realizado um cruzamento das variáveis por meio de tabelas de contingência (crosstabs) para verificar a proporção de adolescentes acometidos por desvios posturais e dor lombar.

As diferenças entre os sexos foram testadas pelo teste $t$ de Student (ângulo lombar) e pelo teste Qui-quadrado $\left(\chi^{2}\right)$ (dor lombar). Foi realizada a regressão de Poisson para verificar a razões de prevalências brutas e ajustadas de desvios posturais associadas ao sexo e à dor lombar. Os valores de significância do modelo bruto foram obtidos pelo teste de Qui-quadrado, pois, de acordo com Hosmer e Leneshow $^{(16)}$, para uma variável permanecer do modelo ajustado ela deve ter $\mathrm{p}<0,25$ no referido teste. Os valores de significância da análise ajustada foram os fornecidos pelo teste de Poisson.

\section{RESULTADOS}

Os dados referentes à dor lombar, considerando as respostas dos alunos, apresentaram uma prevalência de $49,6 \%(n=171)$ no grupo geral, sendo que a frequência, de acordo com os sexos, apontou um acometimento maior pelo grupo feminino, atingindo $53 \%$ das avaliadas, enquanto o grupo masculino apresentou prevalência de $46,2 \%$.

Em relação à frequência da dor na coluna lombar, conforme os dados da Tabela 1, 43,1\% dos adolescentes responderam ter quadros dolorosos ao menos uma vez semanal.

Tabela 1 - Frequência de dor lombar em adolescentes da Rede Federal de Ensino de Florianópolis.

\begin{tabular}{c|c|c|c}
\hline \multirow{2}{*}{ Frequência } & Masculino & Feminino & Geral \\
\cline { 2 - 4 } & $\%$ & $\%$ & $\%$ \\
\hline Todos os dias & 13,2 & 29 & 21,2 \\
\hline Uma vez na semana & 42,7 & 43,5 & 43,1 \\
\hline Uma vez ao mês & 30,9 & 24,6 & 27,7 \\
\hline Raramente & 13,2 & 2,9 & 8 \\
\hline Total & 100 & 100 & 100 \\
\hline
\end{tabular}

Observando a Tabela 1, verifica-se que o grupo feminino apresentou mais que o dobro de casos de dores diárias na coluna lombar que o masculino. As principais respostas relativas aos motivos que levaram ao aparecimento dos quadros dolorosos na coluna lombar, de acordo com os alunos avaliados, estão apresentadas na Tabela 2.

Tabela 2 - Distribuição da frequência das atividades que geraram dor lombar em adolescentes da Rede Federal de Ensino de Florianópolis.

\begin{tabular}{c|c|c|c}
\hline \multirow{2}{*}{ Atividades } & Masculino & Feminino & Geral \\
\cline { 2 - 4 } & $\%$ & $\%$ & $\%$ \\
\hline Atividades domésticas & 5 & 15 & 10 \\
\hline Carregar peso & 19 & 12 & 15,5 \\
\hline Carregar mochila & 10 & 13 & 11,5 \\
\hline $\begin{array}{c}\text { Permanecer sentado } \\
\text { por longos períodos }\end{array}$ & 26 & 25 & 25,5 \\
\hline $\begin{array}{c}\text { Praticar esportes ou } \\
\text { atividades vigorosas }\end{array}$ & 26 & 20 & 23 \\
\hline Flexionar o tronco & 9 & 9 & 9 \\
\hline Estender o tronco & 5 & 6 & 5,5 \\
\hline Total & 100 & 100 & 100 \\
\hline
\end{tabular}


A Tabela 2 mostra que tanto o grupo masculino quanto o feminino apontaram como as principais causas de dor na coluna lombar a permanência por longos períodos na posição sentada e a prática de esportes ou atividades vigorosas.

Quanto aos ângulos lombares gerados a partir da avaliação postural, os dados contidos na Tabela 3 indicam que o grupo feminino apresentou valores angulares superiores da curvatura lombar aos do masculino, sendo esses considerados normais de acordo com a classificação adotada. Por outro lado, a média do grupo masculino $\left(26^{\circ}\right)$, foi classificada como uma redução da angulação (retificação).

Tabela 3 - Valores descritivos do ângulo lombar dos adolescentes da Rede Federal de Ensino de Florianópolis.

\begin{tabular}{c|c|c}
\hline \multirow{2}{*}{ Grupo } & \multicolumn{2}{|c}{ Ângulo lombar $\left(^{\circ}\right)$} \\
\cline { 2 - 3 } & Média & SD \\
\hline Masculino & 26 & 9,09 \\
\hline Feminino & 34 & 8,62 \\
\hline Geral & 30 & 9,60 \\
\hline
\end{tabular}

A análise postural permitiu identificar uma prevalência de desvios na coluna lombar do grupo total equivalente a $53,8 \%(n=155)$, consistindo $90,9 \%(n=141)$ em retificação da coluna lombar e 9,1\% $(n=14)$ em hiperlordose. O comportamento do padrão postural de acordo com os sexos (Figura 1) indica que o grupo masculino apresenta percentuais superiores de alterações posturais $(64,7 \%)$, com $62,8 \%$ dos avaliados apresentando a lombar retificada.

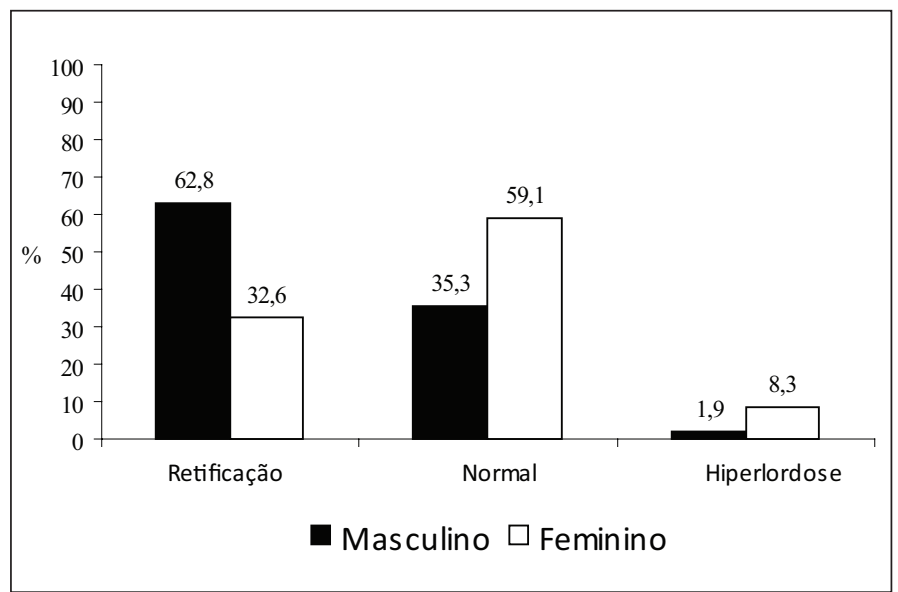

Figura 1 - Distribuição do padrão postural da coluna lombar em adolescentes de acordo com o sexo.

Analisando a Figura 1, observa-se que o grupo feminino possuiu percentuais maiores de angulações normais, consistindo a prevalência de desvios em 40,9\%, estando apenas $8,3 \%$ das avaliadas com a curvatura da lombar acentuada (hiperlordose).

O cruzamento (crosstabs) dos dados da classificação dos desvios posturais com lombalgias indicou que as dores estavam mais associadas aos casos de hiperlordose (Tabela 4).

Tabela 4 - Cruzamento dos valores percentuais (crosstabs) da avaliação postural com dor lombar em adolescentes da Rede Federal de Ensino de Florianópolis.

\begin{tabular}{c|c|c|c|c|c|c}
\hline \multirow{2}{*}{ Ângulo } & \multicolumn{5}{|c}{ Dor lombar } \\
\cline { 2 - 7 } & \multicolumn{2}{|c|}{ Masculino (\%) } & \multicolumn{2}{c}{ Feminino (\%) } & \multicolumn{2}{c}{ Geral (\%) } \\
\cline { 2 - 7 } & Sim & Não & Sim & Não & Sim & Não \\
\hline Normal & 54,5 & 45,5 & 48,7 & 51,3 & 51,1 & 48,9 \\
\hline Retificado & 41,8 & 58,2 & 55,8 & 44,2 & 46,1 & 53,9 \\
\hline Hiperlordose & 33,3 & 66,7 & 72,7 & 27,3 & 64,3 & 35,7 \\
\hline
\end{tabular}

Os dados apresentados na tabela de contingência (Tabela 4) evidenciaram que os quadros de dor estavam mais associados a desvios angulares no plano sagital no sexo feminino $(55,8 \%$ de retificação e $72,7 \%$ de hiperlordose). Foi possível observar ainda que, em geral, a retificação do ângulo lombar teve menor percentual de associação com dor lombar que as demais situações que a coluna esteve exposta.

Como o comportamento das variáveis dor e ângulo lombar se apresentou de forma distinta entre os gêneros, foram testadas as diferenças entre os mesmos, sendo que o ângulo lombar mostrou-se significativamente diferente entre os sexos $(\mathrm{p}<0,001)$, enquanto a variável dor não se apresentou de forma significativa $(\mathrm{p}=0,330)$.

Com o objetivo de analisar a associação de desvios posturais na coluna lombar, controlando possíveis fatores de confusão, foi utilizada a regressão de Poisson, já que o desfecho analisado (ter desvio) apresentou alta prevalência, o que poderia provocar uma superestimativa da magnitude da medida de efeito, odds ratio ${ }^{(17)}$. Foram calculadas as razões de prevalência (RP) brutas e ajustadas, bem como seus respectivos intervalos de confiança de 95\% (IC95\%). A variável dependente, para esta análise, foi dicotomizada em não ter desvio (normal) e ter desvio (Tabela 5).

Os dados da Tabela 5 mostram na análise bruta que o sexo feminino apresentou uma razão de prevalência $37 \%$ menor para desvio na coluna lombar; no entanto, as razões de prevalência para dor lombar não foram significativas $(\mathrm{p}=0,418)$, sendo excluídas do modelo. 
Tabela 5 - Prevalências, razões de prevalências brutas e ajustadas para desvio postural na coluna lombar segundo sexo e dor em adolescentes da Rede Federal de Ensino de Florianópolis.

\begin{tabular}{|c|c|c|c|c|c|}
\hline Variáveis & Prevalências & RP bruta (IC95\%) & p-valor* & RP ajustada (IC95\%) & p-valor** \\
\hline Desvio Postural & 53,8 & - & - & - & - \\
\hline \multicolumn{6}{|l|}{ Sexo } \\
\hline \multirow[t]{2}{*}{ Feminino } & 45,8 & $0,63(0,53-0,74)$ & & $0,69(0,58-0,83)$ & \\
\hline & & & $<0,001$ & & $<0,001$ \\
\hline Masculino & 54,2 & 1 & & 1 & \\
\hline \multicolumn{6}{|l|}{ Dor } \\
\hline \multirow[t]{2}{*}{ Sim } & 49,3 & $0,93(0,80-1,09)$ & & & \\
\hline & & & 0,418 & Excluído do modelo & \\
\hline Não & 50,7 & 1 & & $(p$ bruto $>0,25)$ & \\
\hline
\end{tabular}

*Valor do $p$ não ajustado ( $p$ do Qui-quadrado); **valor do $p$ de tendência ajustado.

\section{DISCUSSÃO}

As altas prevalências de dor lombar entre os adolescentes são consenso na literatura ${ }^{(2,12,18,19)}$, sendo que no estudo de revisão de Balagué et al $l^{(1)}$, os autores encontraram prevalências variando entre 30 e $51 \%$, o que vai ao encontro dos resultados encontrados (Figura 1), indicando que a lombalgia já atinge quase a metade dos adolescentes. Ainda de acordo com o mesmo autor ${ }^{(1)}$, as lombalgias são mais frequentes no sexo feminino, resultado que confirma os achados no presente estudo e vai ao encontro de diversos outros $^{(2,5,6)}$ que evidenciaram essa relação.

A frequência de lombalgia também é algo importante a ressaltar, pois, mais da metade dos adolescentes com esse problema respondeu sentir dor por pelo menos uma vez na semana na coluna lombar (Tabela 1), sendo esse valor superior ao apresentado por Alpalhão e Roballo ${ }^{(20)}$, em seu estudo com 124 adolescentes, que foi de 32,6\%. Se considerar o fato de que, em média, $21 \%$ dos adolescentes deste estudo apresentaram quadros dolorosos diariamente, percebe-se o quanto é importante o diagnóstico precoce de fatores envolvidos nessa problemática, uma vez que a dor pode diminuir a qualidade de vida dos indivíduos.

Nos relatos de dor, as principais atividades apontadas como nocivas foram permanecer muito tempo sentados e praticar esportes ou atividades rigorosas (Tabela 2), o que confirma os achados da literatura, que têm apontado esses fatores como causadores de lombalgias em adolescentes $^{(1,2,5)}$.

A prevalência de desvios posturais sagitais na coluna lombar foi elevada, atingindo 53,8\% dos avaliados.
Nessa perspectiva, Detsch et $a l^{(21)}$, analisando o plano sagital de adolescentes do sexo feminino, encontraram prevalências ainda superiores ao presente estudo, correspondendo a $66 \%$ dos avaliados.

As diferenças dos valores angulares da coluna lombar entre os sexos apresentaram-se significativas $(\mathrm{p}<0,05)$ com valores menores para o grupo masculino $\left(26^{\circ}\right)$, corroborando, dessa forma, com o estudo de Damasceno et $a l^{(22)}$, no qual encontraram diferenças significativas entre os ângulos lombares nos diferentes sexos, assim como em outros componentes da coluna. Para o autor ${ }^{(22)}$, as diferenças parecem estar relacionadas às diferenças existentes entre as medidas dos componentes da curvatura lombar.

Entre outros fatores, essas diferenças também podem estar relacionadas com a distinta constituição estrutural do esqueleto masculino e feminino, na qual o osso da pelve masculina é mais estreito e apresenta uma posição mais vertical do sacro e dos ílios, fato que diminui o ângulo lombar em relação às mulheres ${ }^{(23)}$.

As retificações foram os desvios mais encontrados na coluna lombar (Figura 1), contrariando diversos estudos pesquisados com crianças e adolescentes ${ }^{(14,15,18,21,24-26)}$. Nesses estudos, a análise da lombar no plano sagital apresentou prevalências elevadas de hiperlordose, o que não foi identificado no presente estudo. Essas diferenças podem estar relacionadas aos diferentes métodos utilizados e pela especificidade dos mesmos, pois determinados protocolos de avaliação não permitem avaliar a diminuição das curvaturas da coluna e sim o aumento das mesmas. Além disso, a falta de consenso em relação aos valores de normalidade da lombar tem sido observa- 
da, gerando controvérsias com relação aos limites considerados normais no plano sagital ${ }^{(27)}$, fato que dificulta a comparação entre estudos pelas diferentes metodologias e pontos de cortes adotados.

Outro fator que deve ser levado em consideração é o volume glúteo, que, em alguns casos,pode confundir-se visualmente com o aumento da curvatura lombar, ainda mais quando a avaliação lombar é realizada por meio de instrumentos subjetivos.

Poucos foram os estudos encontrados que apresentaram percentuais de prevalência de retificação na população em geral. Nesse contexto, Blanco et al ${ }^{(19)}$, em um estudo realizado com adolescentes espanhóis, encontraram prevalências de retificação muito inferiores (7\%) às encontradas nos adolescentes de Florianópolis $(47,7 \%)$. Assim como Correia et al ${ }^{(28)}$, em um estudo realizado com crianças da cidade de João Pessoa-PB, identificaram uma prevalência de retificação de $6,8 \%$. No entanto, Giglio e Volpon ${ }^{(26)}$ afirmam que as curvas da coluna aumentam significativamente com a idade, não permitindo comparar dados de crianças com os de adolescentes.

Em relação às prevalências de hiperlordose, os dados do presente estudo foram inferiores a todas as pesquisas encontradas. Os resultados mais próximos do presente estudo foram os de Blanco et al $l^{(19)}$, com percentual de $14 \%$ para esse desvio, e o de Politano ${ }^{(14)}$, em adolescentes de Cacoal-RO, com prevalência de 10,1\%. Martelli e Traebert ${ }^{(25)}$, com adolescentes de uma cidade de Santa Catarina, encontraram prevalências de hiperlordose aproximadamente duas vezes maiores $(20,1 \%)$ que as identificadas no presente estudo. Em uma pesquisa realizada na cidade de Sobral-CE, Mangueira ${ }^{(29)}$ encontrou um percentual de hiperlordose em adolescentes equivalente a $17,5 \%$.

Quando testada a relação entre as variáveis, foi possível identificar que a razão de prevalência de desvios foi superior no grupo masculino, fato que pode estar associado às diferenças estruturais da composição física. As altas prevalências de retificação na coluna dos adolescentes podem estar associadas a fatores nocivos, aos quais os avaliados podem estar expostos.

Considerando que os alunos estão em idade escolar, alguns fatores ligados a hábitos posturais do cotidiano podem estar influenciando no aparecimento de desvios. Sendo assim, as retificações na coluna lombar podem estar associadas, entre outras causas, à posição que os alunos adotam para descanso quando sentados. Nesse contexto, muitas vezes a postura de descanso é uma postura relaxada, na qual o apoio do corpo sobre a cadeira é realizado sobre tuberosidade isquiática e na face posterior do sacro e do cóccix, deixando a pelve em retroversão e a lombar retificada ${ }^{(23)}$, o que pode acabar desenvolvendo encurtamentos musculares, mecanismos compensatórios e lombalgias, quando exposto a longos períodos ${ }^{(30)}$. Cabe ressaltar a importância dos hábitos na postura sentada, uma vez que $25,5 \%$ dos adolescentes avaliados declararam sentir dor na lombar quando permaneciam longos períodos nessa posição (Tabela 2).

Tendo em vista a postura sentada por longos períodos de tempo, pode-se citar Braccialli e Vilarta ${ }^{(31)}$, que explicam que tanto as incidências de desvios quantos os quadros de dor ocorrem, pois a constituição corporal e o modelo biomecânico funcional não foram projetados para permanecer longos períodos sentados e, em função dessa incompatibilidade, resulta as altas prevalências de problemas posturais. Além disso, o ambiente escolar favorece o aparecimento de problemas na lombar, pois a ergonomia do mobiliário é muitas vezes inadequada, com disposições e proporções deficitárias; o peso carregado nas mochilas é excessivo e o transporte do material escolar é realizado de maneira errônea, agravando hábitos posturais inapropriados ${ }^{(31)}$.

A adoção de uma postura incorreta fará com que todo o sistema locomotor participe e se adapte às novas condições mecânicas impostas ${ }^{(30)}$, desenvolvendo, entre outros fatores, um maior gasto energético na manutenção do equilíbrio corporal e na realização das atividades diárias, predispondo os indivíduos à fadiga precoce, o que pode repercutir diretamente em quadros de dor e na qualidade de vida dos indivíduos.

Os resultados obtidos neste estudo, além de contribuir com a literatura da área, apontam a necessidade de uma triagem de problemas posturais em escolares, a qual poderá identificar precocemente esses problemas, assim como hábitos posturais diários que resultem na instalação de problemas permanentes na coluna lombar de adolescentes.

\section{CONCLUSÕES}

Com base nos resultados, pode-se concluir que: a) as prevalências de dores e desvios posturais na coluna lombar dos adolescentes foram elevadas; b) a retificação da lombar foi o desvio postural mais presente, com prevalência superior entre os adolescentes do sexo masculino; c) o ângulo lombar apresentou-se significativamente diferente entre os sexos, sendo maior no grupo 
feminino; d) a prevalência de lombalgias foi superior no grupo feminino; e) independente do sexo, a lombalgia atingiu grande parte dos adolescentes avaliados pelo menos uma vez na semana; f) as principais causas de dor lombar foram a prática de esportes ou atividades vigorosas e a permanência durante longos períodos de tempo na posição sentada; g) as razões de prevalência indicaram que a diminuição do ângulo lombar apresen- tou-se associada com o sexo masculino; e h) a dor lombar não apresentou associação com os desvios sagitais na coluna lombar.

\section{AGRADECIMENTOS}

Nossos agradecimentos ao CAPES e CNPq pelo apoio na realização deste trabalho.

\section{REFERÊNCIAS}

1. Balagué F, Troussier B, Salminen JJ. Non-specific low back pain in children and adolescents: risk factors. Eur Spine J. 1999;8(6):429-38.

2. Andersson GB. Epidemiological features of chronic low-back pain. Lancet 1999;354(9178):581-5.

3. Harreby M, Nygaard B, Jessen T, Larsen E, Storr-Paulsen A, Lindahl A, Fisker I, Laegaard E. Risk factors for low back pain in a cohort of 1389 Danish school children: an epidemiologic study. Eur Spine J. 1999;8(6):444-50.

4. Jones MA, Stratton G, Reilly T, Unnithan VB. Biological risk indicators for recurrent non-specific low back pain in adolescents. Br J Sports Med. 2005;39 (3):137-40.

5. Phélip X. Why the back of the child? Eur Spine J. 1999;8(6):426-8.

6. Marras WS. Occupational low back disorder causation and control. Ergonomics. 2000;43(7):880-902

7. McMeeken J, Tully E, Stillman B, Nattrass C, Bygott IL, Story I. The experience of back pain in young Australians. Man Ther. 2001;6(4):213-20.

8. McGorry RW, Hsiang SM, Snook SH, Clancy EA, Young SL. Meteorological conditions and self-report of low back pain. Spine (Phila Pa 1976). 1998;23(19):2096-102.

9. Evcik D, Yücel A. Lumbar lordosis in acute and chronic low back pain patients Rheumatol Int. 2003;23(4):163-5

10. Christie HJ, Kumar S, Warren SA. Postural aberrations in low back pain. Arch Phys Med Rehabil. 1995;76(3):218-24.

11. Jesus GT, Marinho ISF. Causas de lombalgia em grupos de pessoas sedentárias e praticantes de atividades físicas. Revista Digital [periódico na internet], 2006; 10(92). [Acesso em: 20/06/2007]. Disponível em: www.efdeportes.com/ efd92/lombal.htm.

12. Hakala P, Rimpelä A, Salminen JJ, Virtanen SM, Rimpelä M. Back, neck, and shoulder pain in Finnish adolescents: national cross sectional surveys. BMJ. 2002;325(7367):743.

13. Grimmer K, Dansie B, Milanese S, Pirunsan U, Trott P. Adolescent standing postural response to backpack loads: a randomised controlled experimental study. BMC Musculoskelet Disord. 2002;3:1-10.

14. Politano RC. Levantamento dos desvios posturais em adolescentes de 11 a 15 anos em escola estadual do município de Cacoal-RO [dissertação]. Brasília: Universidade de Brasília, UnB; 2006.

15. Rodrigues PC. Bioestatística. $3^{\mathrm{a}}$ ed. Niterói: EduFF; 2002.

16. Hosmer DW, Leneshow S. Applied logistic regression. New York: Wiley; 1989.

17. Barros AJ, Hirakata VN.. Alternatives for logistic regression in cross-sectional studies: an empirical comparison of models that directly estimate the prevalence ratio. BMC Med Res Methodol. 2003;3:21.

18. Widhe T. Spine: posture, mobility and pain. A longitudinal study from childhood to adolescence. Eur Spine J. 2001;10(2):118-23.

19. Blanco CR, Murillo RA, Velasco RV, Ramirez EM, Sotelo S, Fernández AS. Prevalencia de desviaciones del raquis en escolares del municipio de Talavera de la Reina y sus factores asociados. 2007. [Acesso em: 22/11/2007]. Disponível em: http://sescam.jccm.es/web/gaptalavera/prof-investigacion/desviacionesRaquis.pdf.

20. Alpalhão V, Robalo L. Algias vertebrais nos adolescentes: associação com as actividades de tempos livres auto-reportadas. Rev Essfisionline. 2005;2(1):3-15.

21. Detsch C, Luz AM, Candotti CT, Scotto de Oliveira D, Lazaron F, Guimarães LK, Schinanoski P. Prevalência de alterações posturais em escolares do ensino médio em uma cidade no Sul do Brasil. Rev Panam Salud Publica. 2007;21(4):231-8.

22. Damasceno LH, Catarin SR, Campos AD, Defino, HL. Lordose lombar: estudo dos valores angulares e da participação dos corpos vertebrais e discos intervertebrais. Acta Ortop Bras. 2006;14(4):193-8.

23. Kapandji Al. Fisiologia articular: tronco e coluna vertebral. 5a. ed. Rio de Janeiro: Guanabara Koogan; 2000.

24. Penha PJ, João SM, Casarotto RA, Amino CJ, Penteado DC. Postural assessment of girls between 7 and 10 years of age. Clinic (São Paulo). 2005;60(1):9-16.

25. Martelli RC, Traebert J. Estudo descritivo das alterações posturais de coluna vertebral em escolares de 10 a 16 anos de idade. Tangará-SC, 2004. Rev Bras Epidemiol. 2006;9(1):87-93.

26. Giglio CA, Volpon JB. Development and evaluation of thoracic kyphosis and lumbar lordosis during growth. J Child Orthop. 2007;1(3):187-93.

27. Defino HL, Rodrigues-Fluentes E, Piola FP. Tratamento cirúrgico da cifose patológica. Acta Ortop Bras. 2002;10(1):10-6.

28. Correia PP, Silva GC, Nascimento JF, Lima NM, Sousa MS. Prevalência de desvios posturais em escolares da rede municipal de ensino na cidade de João Pessoa - PB, 2005. [Acesso em 12/04/2007]. Disponível em: www.fef. unicamp.br/ccd/cd/trabalhos/temalivre/P\%E9ricles\%20Paes\%20Barreto\%20 Correia.pdf.

29. Mangueira JO. Prevalência de desvios na coluna vertebral ao exame físico em estudantes de 11 a 16 anos em uma escola do bairro Sinhá Sabóia - Sobral - CE/2004 [monografia]. Sobral: Universidade Estadual do Vale do Acaraú, Sobral/ CE; 2004.

30. Bruschini S, Nery CA. Aspectos ortopédicos da obesidade na infância e adolescência. In: Fisberg M. Obesidade na infância e adolescência. São Paulo: Fundação Editorial BYK; 1995. p. 105-25.

31. Braccialli LMP, Vilarta R. Aspectos a serem considerados na elaboração de programas de prevenção e orientação de problemas posturais. Rev Paul Educ Física. 2000;14(2):159-71. 